\title{
Precision of estimates of phytoplankton growth and microzooplankton grazing when the functional response of grazers may be nonlinear
}

\author{
Geoffrey T. Evans*, Madhu A. Paranjape
}

Department of Fisheries and Oceans, Science Branch, PO Box 5667, St. John's, Newfoundland, Canada A1C 5X1

\begin{abstract}
The analysis of dilution experiments for determining rates of phytoplankton growth and microzooplankton grazing needs to be reconsidered. Zooplankton grazing may saturate at high food concentrations, and therefore experimental data should be fitted with models that include this possibility. Such models will fit the data better; as a consequence, they will reveal some of the uncertainty in the rate estimates that linear analysis conceals. We illustrate this point with simulated and real dilution experiments.
\end{abstract}

\section{INTRODUCTION}

The dilution experiment (Landry \& Hassett 1982) is a technique for separating the net rate of change of a population of phytoplankton or bacteria ('net growth' or 'growth') into the rate of increase due to its own production ('true growth') and the rate of loss due to grazing. In any single incubation these 2 rates are confounded, but they can be separated by performing a series of incubations in which water samples are diluted with different fractions of filtered water. The growth of the phytoplankton population $P$ is assumed to obey the differential equation

$$
\frac{\mathrm{d} P}{\mathrm{~d} t}=(\mu-g X) P,
$$

where $\mu=$ the true, exponential growth rate, $g=$ the specific grazing rate; and $X=$ the fraction of original water in the diluted sample. If we denote the concentration of $P$ in the initial undiluted sample by $P^{*}$, then the initial condition for dilution $X$ is $X P^{*}$. If we denote the final concentration for dilution $X$ by $P_{1}(X)$, then the solution of Eq. (1),

\footnotetext{
- Present address: JGOFS-Büro, Institut für Meereskunde, Düsternbrooker Weg 20, W-2300 Kiel 1, Germany

$$
\frac{1}{t} \ln \left(\frac{P_{1}(X)}{X P^{*}}\right)=\mu-g X,
$$

gives $\mu$ and $g$ as parameters of a linear regression of net growth rate on dilution.

In Eq. (1), the grazing rate $g$ is assumed to be constant, independent of the concentration of $P$ at any time during the incubation. There is considerable interest in relaxing this assumption to take account of phenomena like saturating functional response of the grazers. Tremaine \& Mills (1987) determined that for one set of bacterial experiments they could not reject the hypothesis that grazing is linear; but Tranvik (1989: Fig. 1b) presents some very clean results which, on visual inspection, would appear to fail a lack-of-fit test for a straight line. Gallegos (1989) has proposed a method for analysing data assuming that $g$ depends on the initial value of $P$ but does not change as $P$ changes during any given incubation. He makes measurements at 2 very low dilutions, $X_{1}<X_{2}$, and extrapolates the results to the unattainable dilution $X=0$. If measurements of very dilute samples do not have too large an experimental error, this extrapolation gives an estimate of $\mu$ which makes the least possible commitment to the form of the model, requiring only that the curvature between $X=0$ and $X=X_{2}$ be negligible. This approach is based on the sound principle that we wish to know $\mu$ and $g$, and we should 
not get too distracted by steps along the way, like regression models. It does mean, however, that below $X=X_{2}$ we again have a linear model. Provided one accepts the assumption that $g$ does not change during an incubation, measurement of the undiluted sample then gives a 'model-free' estimate of $\mu-g$.

We want estimates of phytoplankton growth rates in the sea to be precise, which means that we want to be able to reject all but a small range of possible values for them. Our aim in analysing a data set is therefore to show that some possible value $M$ of the growth rate is inconsistent with the data: the smaller the set of $M$ for which we can not say this, the more precise is the estimate. In this regard, an improved ability to handle nonlinear data is not entirely a blessing. If we add to the model a parameter that can describe possibly nonlinear grazing, then the ability to fit the data with a parameter set that includes $M$ improves (Fig 1), and therefore the potential for declaring $M$ inconsistent with the data is diminished. This paper will examine how bad the problem is, with simulated and real data. We shall also discuss whether the 3-point formula of Gallegos, with replicated observations at each of the 3 points, is still the best way of dealing with the problem.

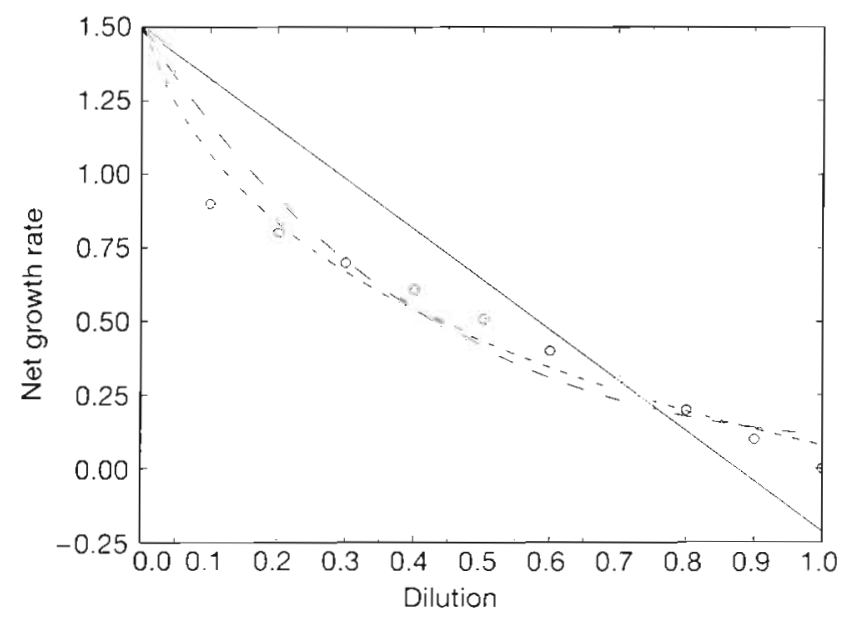

Fig. 1. Data generated from the model of Eq. (2) with $\mu=1(0)$, and the best fitting linear (-), tanh $(--)$ and hyperbolic $(--)$ models constrained to have $\mu=1.5$

\section{THE GRAZING MODEL}

We use a model very like that of Gallegos (1989). In particular, we assume that the grazer population does not change during an incubation, and that the error structure of the growth rates makes analysis by ordinary least squares appropriate. Unlike Gallegos, we shall assume that the grazers respond to the continually varying $P$ during the incubation. This seems more realistic than assuming the grazers 'remember' and respond to $P_{0}$ - especially because nothing in the sea would correspond to the start of an experiment. If growth and grazing are in approximate balance in the undiluted sample then there will not be much difference in practice.

The Gallegos 3-point method uses a linear model below $X_{2}$. If we wish to relax this assumption and accept the possibility of curvature all the way to $X=0$, there is no single obviously correct alternative model. We therefore chose to analyse 2 different forms of grazing function. One is the Michaelis-Menten hyperbola, which gives the equation

$$
\frac{\mathrm{d} P}{\mathrm{~d} t}=\left(\mu-\frac{\alpha X}{1+\beta P}\right) P .
$$

The specific grazing rate at the beginning of the undiluted experiment is

$$
g=\alpha /\left(1+\beta P^{\bullet}\right)
$$

The parameter $\beta$ is not the usual half-saturation parameter; $\beta=0$ gives the linear equation (1) with $g=\alpha$. The other form of grazing reponse is a hyperbolic tangent:

$$
\frac{\mathrm{d} P}{\mathrm{~d} t}=\left(\mu P-\frac{2 \alpha X}{\beta} \frac{e^{\beta P}-1}{e^{\beta P}+1}\right)
$$

Again, the limit as $\beta \rightarrow 0$ is Eq. (1). Because each nonlinear model contains the linear model as a special case, the best nonlinear model must fit at least as well (must have at least as small a sum of squared residuals) as the best linear model. The key difference between the nonlinear models is that the Michaelis-Menten curve has its maximum second derivative at $P=0$ and the hyperbolic tangent has zero second derivative there. The reason why this is important will emerge as we proceed.

\section{LACK-OF-FIT TESTS}

We wish to know which growth rates an experiment enables us to reject. We want a test for rejection that does not depend (much) on the form of the model, and we do not have a fixed alternative hypothesis in mind; so, we proceed as follows. Assume that experiments are replicated at each dilution, so that the 'pure error' variance can be estimated lassuming it is homogeneous, for example that measured growth rates are not more variable at very low dilutions), and standard lack-of-fit tests can be performed (Appendix 1). We 
strongly recommend replication at all dilutions in all dilution experiments: lack-of-fit tests without them are more complicated and less sensitive. Choose a particular value $M$ for $\mu$. Determine the least squares values of $g$ for linear estimation, or $\alpha$ and $\beta$ for nonlinear estimation. Determine whether the best fit model fails the lack-of-fit test. The 'non-rejection' interval for $\mu$ is the set of all $M$ for which the model with an intercept $M$ does not fail the lack-of-fit test. The hypothesis has 2 parts: the model is true and the intercept is $M$. If the the model fails the lack-of-fit test, then either part of the hypothesis may be wrong.

We repeat, we wish to discover which growth rates an experiment enables us to reject. It may often be possible to use other knowledge, such as basic physiological limits, to reject other growth rates and improve the precision, but such analysis is outside the scope of this paper.

\section{SIMULATED DATA}

We wish to state the problems of nonlinearity succinctly; a complete solution would require better knowledge of the error structure of the experiments than is presently available. The data were therefore constructed to indicate how to think about the problem. We started with data from a linear model with model parameters $\mu=1$, and $g=1$, solved the equation for various dilutions, and then added \pm 0.05 to each computed average net growth rate. Thus, the error was exactly the same at every design point, and inferences about the effects of deleting different design points are not affected by different random samples from the error structure. We then determined the most extreme linear, hyperbolic and tanh models that could not be rejected as failing to fit the data generated (Fig. 2). The solutions were determined numerically, using a single Merson step (Appendix 2). We repeated some of the calculations with each of the nonlinear models $(\mu=1, \alpha=1, \beta=1)$.

We present only data for the highest growth rate consistent with the model and data. This corresponds to the quickest saturation, largest $\beta$. The lowest consistent growth rates are hard to interpret. For the hyperbolic model they correspond to negative $\beta$, which means no saturation and infinite grazing at a finite food concentration. For the tanh model, the curves corresponding to $\beta$ and $-\beta$ are the same, so that the lowest consistent growth rate is for $\beta=0$, a linear model.

The results of examining different models and different sets of design points are summarized in Table 1. Because the best fit, and true, value of the growth rate is in all instances $1, \mu-1$ gives a measure of

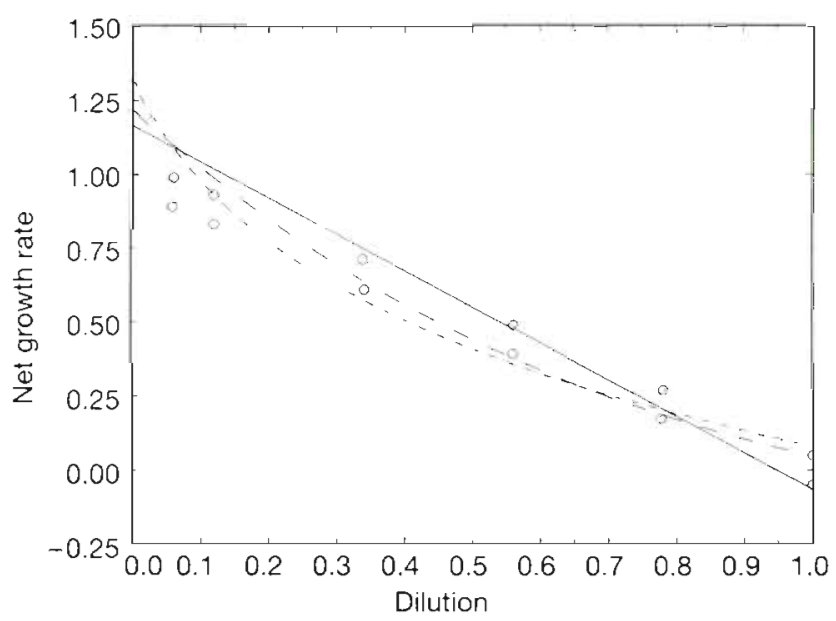

Fig. 2. Noisy data generated from the same model as Fig. 1, and the linear (-), tanh (- - ) and hyperbolic (- - ) models with the highest growth rates that do not fail a lack-offit test for these data

precision (the smaller, the better). We therefore see that, if we do not know that the linear model must be true, asserting that it is true leads us to claim precision about twice as good as can be justified. (And we are assuming here that we know on some other grounds that one of the nonlinear models is correct. If we had to rely on this data set also to choose among nonlinear models, the uncertainty would be much greater.) The value of observations at low dilutions is seen when we delete observations at the smallest design point (third column of Table 1), especially if the hyperbolic model best describes the grazing response. What happens, then, if we adopt the plan of Gallegos (1989) and make measurements only at 2 very dilute samples plus the undiluted? The 3-point method of Gallegos gives 4 possible values for $\mu$ : $0.85,0.95,1.05$ and 1.15 , whose range is the same as linear estimation would suggest. However, the limits from nonlinear estimation expand considerably (last column of Table 1).

Table 1 The largest value of $\mu$ not rejected by a lack-of-fit test. 'All points' column: all the data shown in Fig. 2 are included in the analysis. 'Lowest point deleted' column: the observations for dilution 0.06 are omitted from the analysis. '3-point, 4 replicates' column: only the observations for dilutions $1,0.12$ and 0.06 are included in the analysis, and each is treated as if it had occurred in each of 2 independent measurements, so that there are as many measurements as in the 'all points' column

\begin{tabular}{|lccc|}
\hline Estimation & All points & $\begin{array}{c}\text { Lowest point } \\
\text { deleted }\end{array}$ & $\begin{array}{c}\text { 3-point } \\
\text { 4 replicates }\end{array}$ \\
\hline Linear & 1.16 & 1.21 & 1.13 \\
Tanh & 1.22 & 1.33 & 1.41 \\
Hyperbolic & 1.33 & 1.67 & 1.78 \\
\hline
\end{tabular}


We computed the range of initial grazing rates using Eq. (4) for the best-fit values of $\alpha$ and $\beta$, and also as Gallegos (1989) would calculate them on the assumption that the specific grazing rate was constant during the incubation. For this set of parameters, the difference between the 2 grazing models is negligible compared to the uncertainty.

Finally (Table 2) if the data are generated from a nonlinear model then the precision claimed by the linear model is even more exaggerated. The improvement in precision for linear estimates of data generated by a nonlinear model is only apparent: what is really being rejected is the linear model, not the parameter values.

Table 2. The largest value of $\mu$ not rejected by a lack-of-fit test, comparing linear and nonlinear estimation of linear and nonlinear models. 'Linear' column is the same as 'all points' in Table $1_{i}$ for the 'tanh' (resp. 'hyperbolic') column, the data (not shown) are generated from the tanh (resp. hyberbolic) model with the nonlinear parameter $\beta=1$

\begin{tabular}{lccc|}
\hline Estimation & Linear & $\begin{array}{c}\text { Model } \\
\text { Tanh }\end{array}$ & Hyperbolic \\
\hline Linear & 1.16 & 1.11 & 1.10 \\
Tanh & 1.22 & 1.25 & 1.65 \\
Hyperbolic & 1.33 & 1.84 & 3.2 \\
\hline
\end{tabular}

\section{REAL DATA}

We used experiments performed on board CSS 'Baffin' during the North Atlantic Bloom Experiment (NABE) of the Joint Global Ocean Flux Study, in May 1989. In this paper we shall treat phytoplankton as represented by chlorophyll measurements; a more sophisticated pigment analysis, with more details of the experimental procedure, will be reported elsewhere

Fig. 3 shows the data, and the most extreme linear and nonlinear fits, for an experiment begun on 1 May near $40^{\circ} \mathrm{N}, 47^{\circ} \mathrm{W}$. The linear and nonlinear ranges do not differ much. The highest acceptable growth rate is smaller for the tanh model than for the linear model because, with more parameters, a nonlinear model has stricter rejection criteria. The data are noisy, and the low measurements at $X=0.76$ cast doubt on any model - especially one with more parameters that ought to be able to do better.

Fig. 4 shows the data, and the most extreme linear and nonlinear fits, for an experiment begun on 9 May near $44^{\circ} \mathrm{N}, 41^{\circ} \mathrm{W}$. The data are less noisy, and the eye of faith might see a slight concavity. Now both nonlinear models embrace growth rates higher than the linear model would accept. If we accept the linear model, then we could be claiming a precision more than twice as good as we are entitled to.

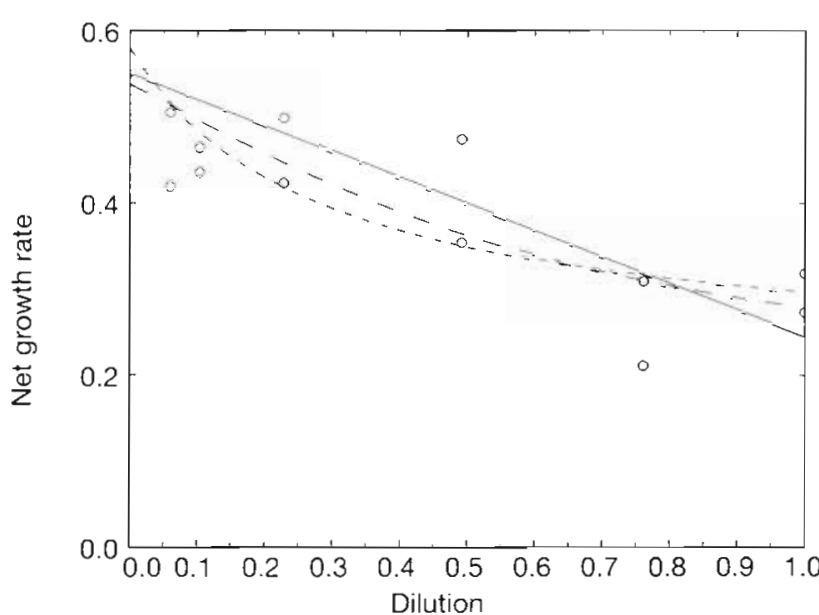

Fig. 3. Data collected in the 1 May 1989 NABE measurements. Lines as in Fig. 2

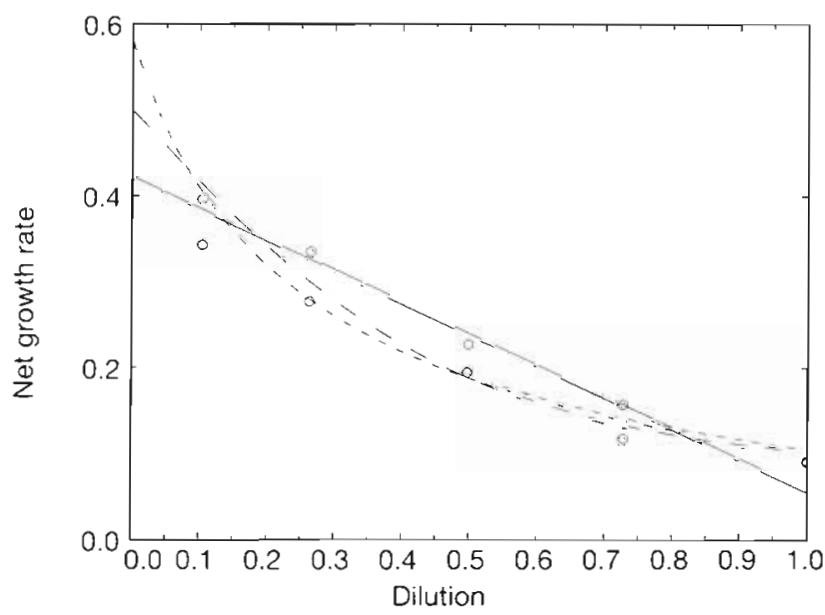

Fig. 4. Data collected in the 9 May 1989 NABE measurements. Lines as in Fig. 2

\section{DISCUSSION}

Two basic issues underlie this work: must we accept the possibility of curvature below the most dilute sample, and therefore a lower precision in growth rate estimates; and if so, how do we estimate the curvature? To the first question we give an unequivocal yes; we cannot resolve the second, but merely call attention to its importance and to the need for more work.

A common attitude in statistics is that data are fundamental, parameters of a model just provide a convenient way to summarize them, and one should accept the simplest model that cannot be shown to be inconsistent with the data. Tremaine \& Mills (1987) adopt this position. However, we would argue that dilution experiments should not be treated in this way. 
For them, the scientifically fundamental quantities are the parameters, true growth and grazing, that describe what was actually happening in the sea. The data at intermediate dilutions are artificial and of no intrinsic interest; the issue is not how they can be economically described, but how they might have been generated. A linear model that we cannot reject has no firmer standing than any of saturating models that we cannot reject. The possibility of nonlinear grazing response is so widely believed, and so important for the behaviour of population dynamics models, that we must consider it even when a linear model cannot be rejected. The main result of considering it is not to enable us to fit data better (although it will do that) but to force us to recognize that the range of parameters that can produce a good fit (according to lack-of-fit criteria) is wider than had been thought, that the certainty about $\mu$ and $g$ is much less than linear analysis would erroneously suggest.

The 2 nonlinear models behave differently because of their second derivatives at the origin. Below the smallest measured concentration there are no data to constrain how the grazing response bends, but the tanh model asserts that it stops bending as the concentration goes to zero, whereas the hyperbolic model asserts that the bending continues to increase. It is appropriate to ask which model is better, but neither the theory nor the data presented here can help us with that question. The theory only starts after a choice of response function has been made, and both the tanh and hyperbolic models can be made to fit the NABE data acceptably according to the lack-of-fit tests. What we have revealed that was not previously apparent is not the answer to the question, but the importance of determining an answer.

The best way to estimate the second derivative at extreme dilutions depends on whether we have a trusted theory of grazer functional response. If we have, then measurements at intermediate $X$ contain information about curvature at low $X_{1}$ and it is best to use them. We can see this by observing how poorly the 3-point, 4-replicate method, which ignores information at intermediate dilutions, performed. If we have no trusted theory, then we are reduced to numerical estimation of the second derivative. This involves tradeoffs between noise and third derivatives, along the lines suggested by Press \& Teukolsky (1991) for estimating slopes but more difficult and less rewarding. We have no magic answer, and must face the fact that dilution experiments have not brought us as far as had been hoped.

A more detailed consideration of experimental design, including all we can learn about sources of error, would be useful. A complete study would also include how to incorporate other knowledge, like physiological limits on growth, to increase the number of rejected possibilities. The best design of course depends on the aim of the experiment. We have assumed here that the aim was simply to report growth and grazing rates; if we wish to know the shape of the whole functional response curve then the design points should be spread out more. (In this case one might prefer to use cultured, clonal populations to reduce noise.) The use to which parameters will be put is another consideration. For example, if we believe (from sensitivity analyses of simulation models, for example) that the behaviour of grazers at low food densities (possible grazing thresholds, for example) is more important to the overall dynamics of marine populations than the precise level of saturation at high food densities, then we might prefer a weighted fit that attached more importance to fitting the low end well.

The estimated grazing rate is just the difference of the true growth rate and the undiluted net growth rate. Because the undiluted net growth rate can be measured without having to extrapolate, it contributes little extra to the uncertainty which is mainly in the estimate of true growth rate. Therefore our conclusions about the precision of growth estimates apply basically unchanged to grazing rate estimates.

Throughout this analysis, we have assumed that only $P_{0}$ and $P_{\uparrow}$ are measured. In fact, Tremaine \& Mills (1987) and Tranvik (1989) estimated average growth rates from several measurements during the time course of an incubation. When grazing response is nonlinear, more use can be made of intermediate measurements, because as $P$ changes during the incubation the degree of saturation changes and we get information about the separate parameters. It is tempting to try and fit all of the raw data on concentrations from all dilutions with a single set of parameters, although correlated errors will cause problems.

Microzooplankton grazers have generation times not much different from the cells they are eating, and therefore their populations can also be expected to change during the incubation. If we try to allow for this without any extra measurements, we add at least one more parameter to help us fit the data, and thus do even more horrible things to the precision of parameter estimates. The same happens if we try to fit a grazing curve that is initially concave upwards, reflecting a grazing threshold or the like, and then saturates.

Acknowledgements. Comments by Charles Gallegos have greatly improved this paper. In particular, he pointed out how special the Michaelis-Menten hyperbola is in having such high curvature near the origin. 


\section{APPENDIX 1}

\section{Lack-oi-fit testing}

This appendix is basically a copy of Section 2.1.5 of Seber \& Wild (1989). Given replicated observations $y_{i k}$ at each design point $x_{j}$, form the sample mean $\bar{y}_{i}$ at each $x_{i}$, and compute the pure error sum of squares

$$
Q=\sum_{l} \sum_{k}\left(y_{l k}-\bar{y}_{l}\right)^{2}
$$

Given a model with a predicted value $\eta_{i}$ at each $x_{j}$ compute the residual sum of squares

$$
Q_{H}=\sum_{i} \sum_{k}\left(y_{i k}-\eta_{i}\right)^{2}
$$

If there are $N$ observations in total, $n$ design points, and $p$ model parameters to be estimated, then the ratio

$$
F=\frac{Q_{H}-Q}{Q} \frac{N-n}{n-p}
$$

is approximately (exactly for models that are linear in the parameters) distributed as $F_{\mid n-p, N-n]}$. The model fails the lack-of-fit test at the 0.05 level if $F$ is greater

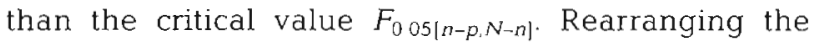
equation, there is lack-of-fit if

$$
Q_{H}>\left(1+\frac{n-p}{N-n} F_{0.05[n-p, N-n]}\right) Q
$$

For example, if $N=12, n=6$, and $p=1$, then we have lack of fit if the residual sum of squares is more than 4.7 times as large as the pure error sum of squares. Remember that $p$ is the number of free parameters; e.g. if $\mu$ is fixed and we are using linear estimation, then $p=1$.

\section{LITERATURE CITED}

Fox, L. Mayers, D. F. (1987). The numerical solution of ordinary differential equations. Chapman and Hall, London

Gallegos, C. L. (1989). Microzooplankton grazing on phytoplankton in the Rhode River, Maryland: nonlinear feeding kinetics. Mar. Ecol. Prog. Ser. 57: 23-33

Landry, M. R., Hassett, R. P. (1982). Estimating the grazing impact of marine microzooplankton. Mar. Biol. 67: $283-288$

Press, W. H., Teukolsky, S. A. (1991). Numerical calculation of

This article was presented by Drs E. and B. Sherr, Corvallis, Oregon, USA

\section{APPENDIX 2}

\section{Numerical approximation to the solution}

lo approximate the value $P_{1}$ of the solution of the differential equation

$$
\frac{\mathrm{d} P}{\mathrm{~d} t}=f(P)
$$

at Time 1 (in some appropriate units), given the initial condition $P=P_{0}$ at Time 0 , we propose to use a single integration step, and are therefore not concerned with good behaviour as the step size changes; but we do want an estimate of the local error. We therefore use the formula of Merson (e.g. Fox \& Mayers 1987, p.68):

$$
\begin{aligned}
& k_{1}=f\left(P_{0}\right) \\
& k_{2}=f\left(P_{0}+\frac{1}{3} k_{1}\right) \\
& k_{3}=f\left(P_{0}+\frac{1}{6} k_{1}+\frac{1}{6} k_{2}\right) \\
& k_{4}=f\left(P_{0}+\frac{1}{8} k_{1}+\frac{3}{8} k_{3}\right) \\
& k_{5}=f\left(P_{0}+\frac{1}{2} k_{1}-\frac{3}{2} k_{3}+2 k_{4}\right) \\
& P_{1} \approx P_{0}+\frac{1}{6} k_{1}+\frac{2}{3} k_{4}+\frac{1}{6} k_{5}
\end{aligned}
$$

which has a local error of approximately

$$
\left(2 k_{1}-9 k_{3}+8 k_{4}-k_{5)} / 30 .\right.
$$

Local truncation error was never a problem for the net growth rates we considered.

derivatives. Computers in Physics 5: 68-69

Seber, G. A. F., Wild, C. J (1989). Nonlinear regression. Wiley, New York

Tranvik, L. J. (1989). Bacterioplankton growth, grazing mortality and quantitative relationship to primary production in a humic and a clearwater lake. J. Plankton Res. 11: $985-1000$

Tremaine, S. C., Mills, A. L. (1987). Test of the critical assumptions of the dilution method for estimating bacteriovory by microeucaryotes. Appl. environ. Microbiol. 53: $2914-2921$

Manuscript first received: October 20, 1990 Revised version accepted: January 27, 1992 